\title{
Positron annihilation and tribological studies of nano-embedded Al alloys
}

\author{
JERZY DRYZEK ${ }^{1, *}$, KRZYSZTOF SIEMEK $^{1}$, KRZYSZTOF ZIEWIEC $^{2}$ and HENK SCHUT ${ }^{3}$ \\ ${ }^{1}$ Institute of Nuclear Physics Polish Academy of Sciences, ul. Radzikowskiego 152, 31-342 Kraków, Poland \\ ${ }^{2}$ Institute of Technology, Faculty of Mathematics, Physics and Technical Science, \\ Pedagogical University of Cracow, ul. Podchorążych 2, PL-30-084 Kraków, Poland \\ ${ }^{3}$ Interfaculty Reactor Institute, Delft University of Technology, Mekelveg 15, NL-2629 JB Delft, The Netherlands
}

MS received 12 January 2015; accepted 30 March 2015

\begin{abstract}
Positron annihilation studies of aluminium alloys with nanodispersions of insoluble elements, i.e., $\mathrm{In}, \mathrm{Sn}, \mathrm{Pb}$ and $\mathrm{Au}$ were reported. The alloys were obtained using a rapid solidification process. For all alloys, except that with Au, the average diameter of nanoparticles in aluminium matrix was $100 \mathrm{~nm}$, and variance of the size distribution was above $50 \mathrm{~nm}$. Positron annihilation studies reveal the presence of monovacancies or divacancies, which were located at the interface between nanoparticles and the matrix. In the as-cast reference pure aluminium sample as well as the aluminium and gold alloy dislocations were identified as well. The isothermal annealing of the obtained alloys and measurement of the annihilation characteristic, i.e., $S$-parameter, allow us to determine the activation energy of grain boundary migration, which for the alloys was higher by the factor of four than for the reference sample. The measurements of friction parameters for the alloys confirmed the results reported by the other authors that, the friction coefficient was lower by the factor of about two and the specific wear rate was by the factor of about fifty higher than the reference sample. The present study confirmed the attractive positron affinity of the nanoparticles of In, Sn, Pb and Au compared to aluminium matrix.
\end{abstract}

Keywords. Positron annihilation; aluminium alloys; nanoparticles.

\section{Introduction}

There is growing interest in binary immiscible systems as potential objects of different studies and applications. Such systems have been applied as bearing materials long time ago (see the review in ref. 1). For instance Al-Sn system is used as bearing material, due to a good combination of strength and surface properties, which can be an alternative to environmentally hazardous $\mathrm{Pb}-\mathrm{Sb}-\mathrm{Sn}$ system (Babbitt) commonly used. Later the $\mathrm{Al}-\mathrm{Pb}$ alloys were considered as a cheaper alternative. ${ }^{2}$ The modified impeller mixing and chill casting technique were applied for the preparation of these alloys. Their microstructure exhibits the particles and patches in interdendritic region of aluminium. However, the rapid solidification process is also used. This process allows to produce the microand nano-scaled dispersoids in the alloys. Bhattacharya et $a l^{3-6}$ established the good tribological properties under mild wear condition of the nano-embedded Al alloys obtained in such a process. The effect of particle's size on the tribological properties is most interesting. ${ }^{5}$ It was found that the alloy samples with nanosized dispersions of $\mathrm{Pb}$ particles exhibit lower friction coefficient com-

\footnotetext{
*Author for correspondence (jdryzek@gmail.com)
}

pared to micron-sized dispersed particles. This is due to the fact that smearing of $\mathrm{Pb}$ film is more homogeneous in the nanodispersed alloy. The adhesive-type wear mechanism was observed by Pathak and Mohan ${ }^{2}$ and confirmed by Bhattacharya and Chattopadhyay. ${ }^{5}$ Significant reduction of the friction coefficient and wear was also obtained in $\mathrm{Al}-\mathrm{In}^{7}$ and $\mathrm{Al}-\mathrm{Bi}^{3}$ alloys. Also the bimetallic dispersoids of $\mathrm{Sn}$ and $\mathrm{Pb}$ were produced using this process. ${ }^{8}$ The orientation relation of nanoparticles with the aluminium matrix was shown as well. The rapid solidification process induces that the alloys are far from the equilibrium state and contain many defects, which can affect the properties as well. The aim of the presented paper is to perform the positron annihilation studies of the immiscible systems of aluminium alloys obtained in this process. The positron techniques are suitable for studies of defects at the atomic level (see for instance ref. 9).

Numerous reports have been published on the Al alloys' studies using positron annihilation methods (see for instance ref. 10). Due to their high sensitivity they are suitable for detecting open-volume sites in defect solids. The open volume defect occur as a result of structural changes in age hardening, misfit interfaces and grain boundaries or any deformation processes, for instance fatigue or plastic deformation. Another interesting feature 
detected by the positron slow beam technique and coincidence Doppler broadening spectroscopy is the high sensitivity to positron trapping at $\mathrm{Au}$ and $\mathrm{Sn}$ ultra thin layers in Al. ${ }^{11,12}$ This phenomenon arises from the high positron affinity to the small clusters of heavy elements like Au, $\mathrm{Sn}$ and $\mathrm{Pb}$ related to $\mathrm{Al}$. Our studies intended to confirm these results using the conventional positron technique and the ${ }^{22} \mathrm{Na}$ source for the Al alloys with nanoparticles. To broaden the subject of our studies, the Al-In and Al$\mathrm{Au}$ alloys are also included and the thermal stability of the obtained alloys in the isochronal annealing experiment was tested. In our research the microhardness, scanning electron microscopy (SEM) and X-ray diffraction (XRD) measurements as a complementary are also applied.

\section{Experimental}

\subsection{Sample preparation}

Appropriate amounts of metals In (99.99\%), Sn (99.9\%), $\mathrm{Pb}(99.9 \%)$ and $\mathrm{Au}(99.9 \%)$ were melted with $\mathrm{Al}$ (99.99\%) in quartz crucible in Ar atmosphere using the RF technique. The melted metals were rapidly solidified through $0.5 \mathrm{~mm}$ orifice quartz nozzle on to the surface of a polished $\mathrm{Cu}$ wheel rotating at a speed of $22 \mathrm{~m} \mathrm{~s}^{-1}$, using an Ar gas overpressure of $15-20 \mathrm{kPa}$. The ribbons of thickness of about $50-80 \mu \mathrm{m}$ and $1-2 \mathrm{~cm}$ long were obtained. The composition of obtained alloys is as follows: Al-In5.7, Al-Sn5.9, Al-Pb11.2 and Al-Au1, where digits represent in per cent the approximate weight ratio of a metal determined using the Rutherford backscattering spectrometry (RBS) technique. The spun ribbons were pressed using hydraulic press with pressure of $20 \mathrm{MPa}$ to form dense pellets, $10 \mathrm{~mm}$ in diameter and $3 \mathrm{~mm}$ thick used for further measurements.

\subsection{Positron measurement details}

In our studies we used three positron techniques, the positron lifetime measurements (PALS), Doppler broadening of annihilation line (DB), and its coincidence version called coincidence Doppler broadening (CDB), characterized by significant reduction of background below the annihilation line.

For the positron lifetime measurement, fast-fast positron lifetime spectrometer with $\mathrm{BaF}_{2}$ scintillators was used. The time resolution full-width half-maximum (FWHM) was equal to 260 ps. The positron source, i.e., ${ }^{22} \mathrm{Na}$ enveloped in the $7 \mu \mathrm{m}$ Kapton foil was located between the worn surfaces of the two identical samples, and this sandwich was positioned in front of the scintillator detectors of the positron lifetime spectrometer. The positron lifetime spectrum was measured to obtain more than $2 \times 10^{6}$ counts. The average implantation depth, which is the reciprocal value of the linear absorption coefficient, ${ }^{13}$ for positrons emitted from ${ }^{22} \mathrm{Na}$ isotope in $\mathrm{Al}$ is ca. $90 \mu \mathrm{m} .{ }^{13}$ This value indicates the depth from the measured surface, which is scanned by positrons in each measurement. About $75 \%$ of emitted positrons annihilate in the layer of such a thickness as simulated by the LYS-1 computer code. ${ }^{14}$

Measurements of the DB give valuable results, which were performed using a HpGe detector. The energy resolution of the $\mathrm{HpGe}$ detector system was equal to 1.38 at $511 \mathrm{keV}$. As in the case of positron lifetime measurements, the positron source was sandwiched between two samples. For Doppler broadening measurements, ${ }^{68} \mathrm{Ge} /{ }^{68} \mathrm{Ga}$ positron emitter was applied, enveloped in $7 \mu \mathrm{m}$ Kapton foil, for which the end point energy is $1.88 \mathrm{MeV}$ and in this case the average positron implantation range in the studied aluminium equals to $470 \mu \mathrm{m}$. The ${ }^{68} \mathrm{Ge} /{ }^{68} \mathrm{Ga}$ emitter is a convenient source, because the peak to background ratio for annihilation line is better by a factor of 4 than that for ${ }^{22} \mathrm{Na}$ emitter.

The CDB measurements were performed using two $\mathrm{HpGe}$ detectors separated by $1 \mathrm{~m}$ from each other. The samples were located in the middle between the detectors. The detectors measured the energies of two annihilation photons which were stored in the $2 \mathrm{D}$ map. The sum of the counts in the diagonal region of the map allows us to obtain the DB spectrum with reduction of the background. About $4 \times 10^{6}$ counts were accumulated for each spectrum. The peak background ratio achieved is about $10^{-5}$.

\section{Results and discussion}

\subsection{Microscopy results}

The typical low-magnification electron micrographs of the rapidly solidified sample containing $\mathrm{In}, \mathrm{Sn}, \mathrm{Pb}$ and $\mathrm{Au}$ nanoparticles embedded in Al matrix are shown in figure 1. The micrographs display a large dispersion of almost spherical particles. In addition, one can observe the presence of both large and fine particles. One can also notice that in the case of Al-In5.7 the largest particles are located in the vicinity of grain boundaries, as it is seen in figure 2 .

The particle size distribution was obtained from the projected SEM images using ImageJ software. ${ }^{15}$ The diameter distribution histograms are depicted in figure 3. They reveal long tails indicating the presence of particles with large diameter, which was also seen by Bhattacharya and Chattopadhyay. ${ }^{3}$ The average diameter and the standard deviation of the distribution, i.e., for all studied alloys, are gathered in table 1. For Al-In5.7 the average diameter is the lowest, about $83 \mathrm{~nm}$. Similar dependence is noticed for the variance of the distribution. For the alloy Al-Au1, the size of the Au particles was too small to make proper statistics from SEM images. 

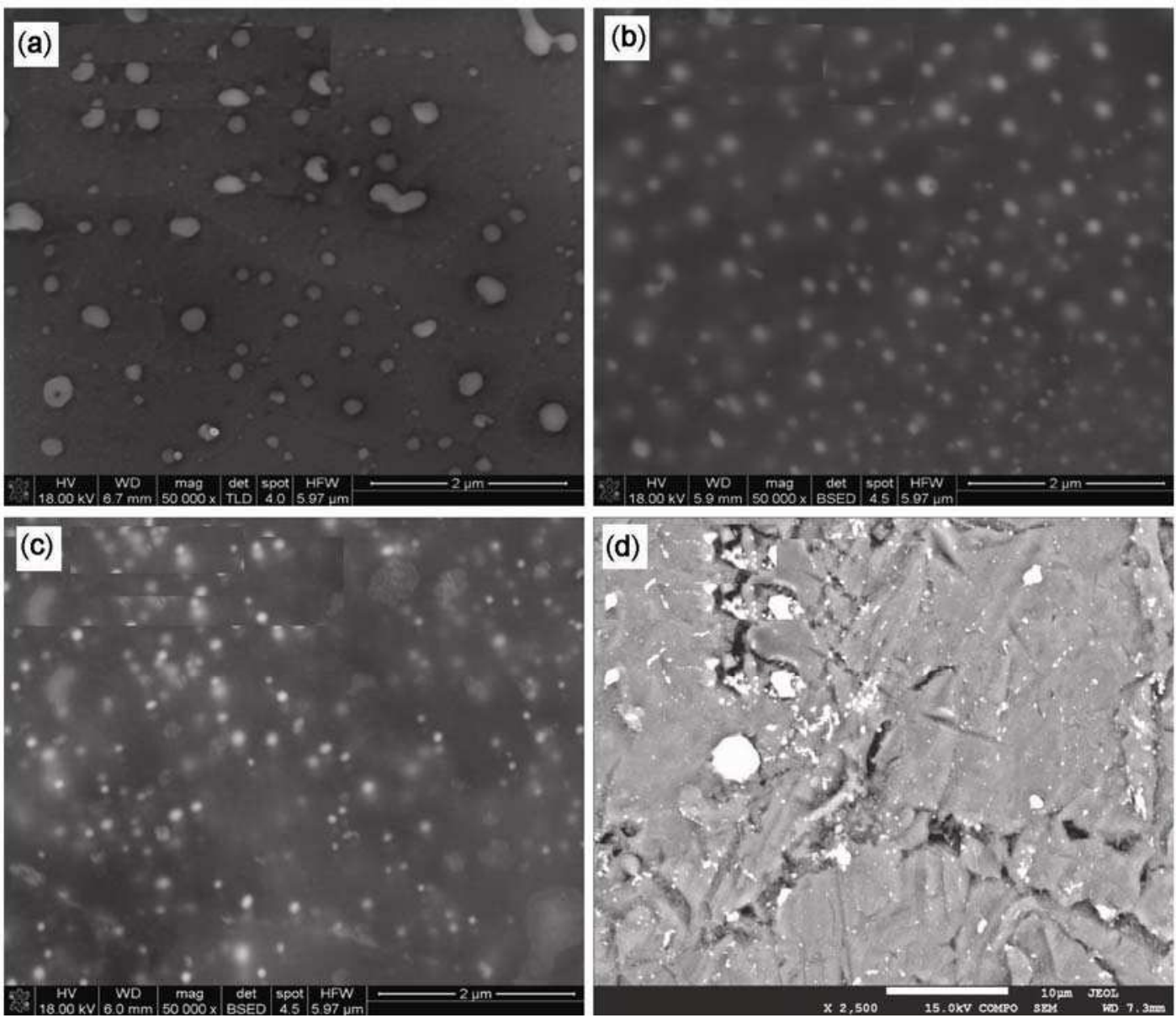

Figure 1. SEM micrographs of the alloys: (a) Al-In5.7, (b) Al-Sn5.9, (c) Al-Pb11.2 (10) and (d) AlAul obtained by the rapid solidification process. Embedded nanoparticles of the alloys metals are visible.

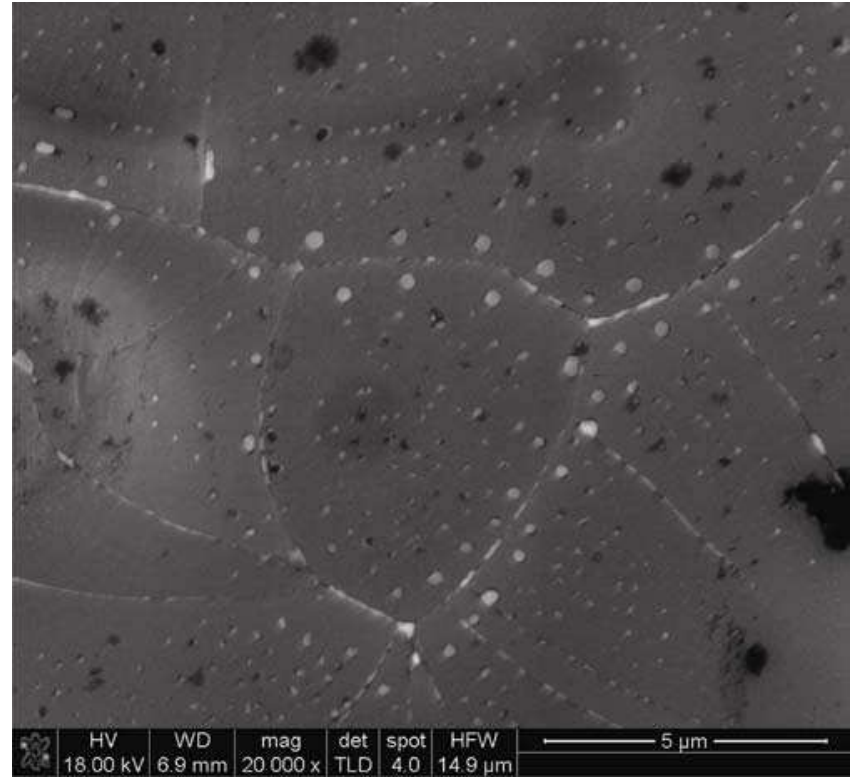

Figure 2. SEM micrograph for the Al-In5.7 alloy, which shows the distribution of In nanoparticles on or close to the grain boundary.

\subsection{XRD results}

XRD measurements reveal that despite of rapid solidification both Al matrix and the nanoparticles exhibit crystalline structure. The well-defined peaks originated from the crystalline structure are detected (figure 4). However, due to the small amount, peaks from Au are not visible in the XRD pattern.

\subsection{Friction properties}

Friction properties of the obtained alloys were measured using the conventional pin-on-disc set-up. The pin was the pellet made of the alloy and the disc made of the martensitic steel (SW18), with measured Vickers Hardness under $100 \mathrm{~g}$ load equals to $670 \mathrm{HV}$. In table 1 the obtained values of the friction coefficient and the specific wear rate are gathered. For comparison we also carried out measurements for the reference sample, which is the ascast pure aluminium sample obtained in the same process. This sample contains only defects that are generated during rapid solidification and no other particles. The friction 

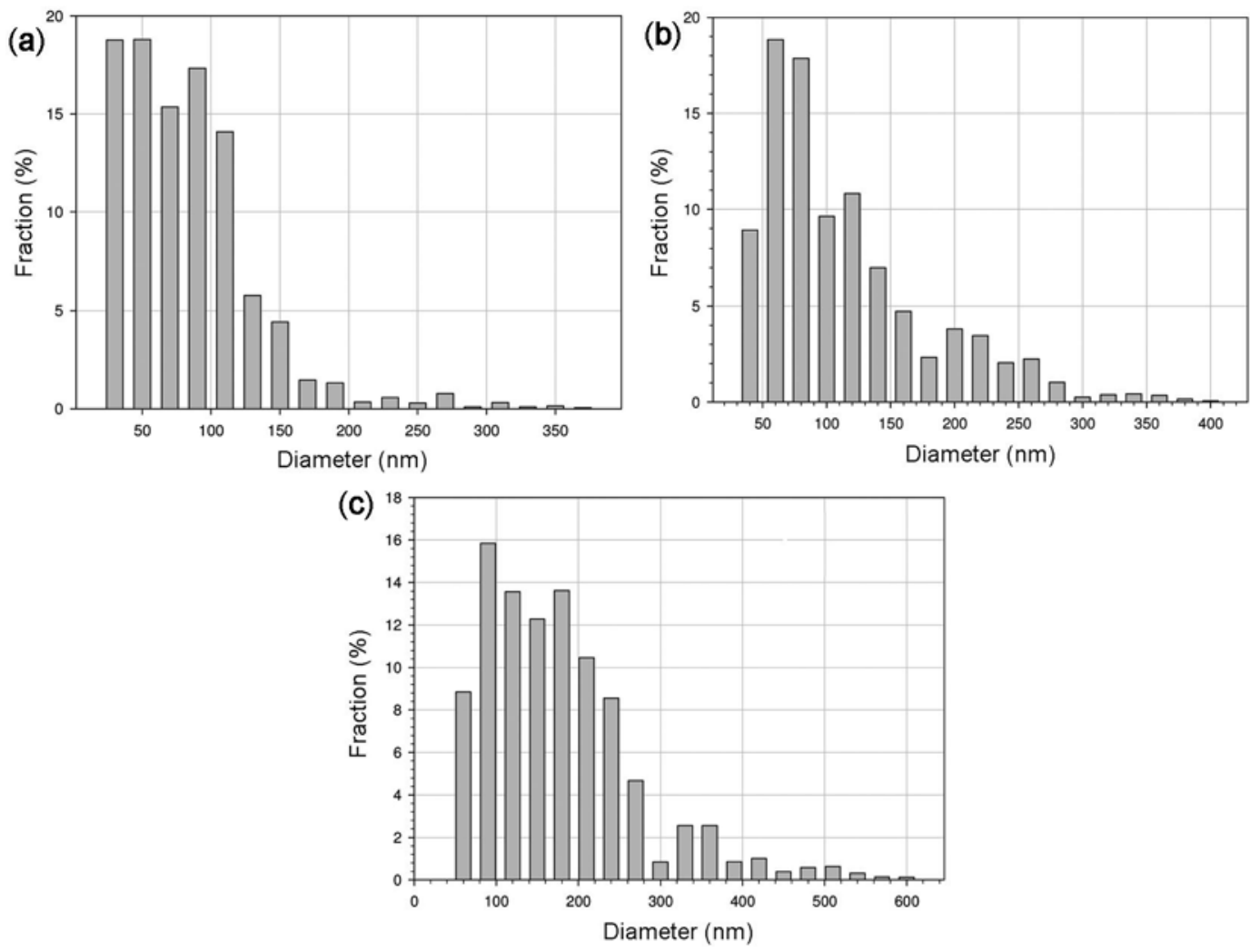

Figure 3. Diameter distribution histograms of the nanparticles obtained from the SEM micrographs using ImageJ software for the (a) Al-In5.7, (b) Al-Sn5.9 and (c) Al-Pb11.2 alloys.

Table 1. The values of the average diameter of the particles dispersed in the Al matrix for selected alloys. The average particle diameter $D$ and variance $\sigma_{\mathrm{D}}$ were obtained from distribution depicted in figure 3 . The values of the tribological parameters, i.e., the friction coefficient $f$ and specific wear rated SWR and Vickers microhardness HV are presented. The values of the positron lifetime (PLT) in the last column are obtained after obtaining the alloys. $Q$ represents the value of the activation energy for grain boundary migration obtained from the fit of the diffusion trapping model to the experimental points for the alloy in the cumulative isochronal annealing.

\begin{tabular}{lccccccc}
\hline Alloy & & \multicolumn{5}{c}{$\mathrm{SWR}$} \\
& $D(\mathrm{~nm})$ & $\sigma_{\mathrm{D}}(\mathrm{nm})$ & $f$ & {$\left[10^{-12} \mathrm{~m}^{3} \mathrm{mN}^{-1}\right]$} & $\mathrm{HV}$ & PLT $(\mathrm{ps})$ & $Q(\mathrm{eV})$ \\
\hline Al-In5.7 & 83 & 48 & $0.38 \pm 0.05$ & 0.058 & $71.6 \pm 5.5$ & $256.9 \pm 0.5$ & $2.12 \pm 0.4$ \\
Al-Sn5.9 & 108 & 64 & $0.39 \pm 0.05$ & 0.099 & $41.3 \pm 3.8$ & $253.7 \pm 0.5$ & $1.12 \pm 0.2$ \\
Al-Pb11.2 & 173 & 92 & $0.40 \pm 0.05$ & 0.084 & $39.2 \pm 3.1$ & $257.2 \pm 0.5$ & $1.43 \pm 0.3$ \\
Al-Au1 & - & - & $0.42 \pm 0.05$ & 0.066 & $59.3 \pm 3.8$ & $224.1 \pm 0.5$ & $1.73 \pm 0.4$ \\
Al-as-cast & - & - & $0.82 \pm 0.05$ & 3.295 & $28.6 \pm 2.6$ & $229.7 \pm 0.5$ & $0.65 \pm 0.2$ \\
\hline
\end{tabular}

coefficient for the alloys is almost constant and equal to 0.4 , which is about half of the value for the aluminium sample. Bhattacharya and Chattopadhyay reported the decrease of the friction coefficient with the increase of the applied load. ${ }^{3}$ In our studies we tested this and found no significant change of the friction coefficient in the range of loads between 0.5 and $200 \mathrm{~N}$. However, the specific wear rate varies depending on the alloy. This rate is defined as worn volume per unit sliding distance per unit load. Al-In5.7 alloys has lower value, i.e., $5.8 \times 10^{-14}$ $\mathrm{m}^{3} \mathrm{mN}^{-1}$, and highest for the Al-Sn5.9 alloy, i.e., $9.9 \times$ $10^{-14} \mathrm{~m}^{3} \mathrm{mN}^{-1}$. These values are much smaller in comparison to the reference aluminium sample, for which the wear coefficient is about 45 times higher, i.e., $3.3 \times$ $10^{-12} \mathrm{~m}^{3} \mathrm{mN}^{-1}$. The measurements in the identical conditions of commercial alloy, AlSi $13 \mathrm{Mg} 1 \mathrm{CuNi}$, gave the specific wear rate of $6.6 \times 10^{-14} \mathrm{~m}^{3} \mathrm{mN}^{-1}$. Thus, it is lower than for the Al-Sn5.9 and Al-Pb11.2 alloys. The significant reduction of the wear rate for the alloys was reported already by other authors, see ref. 3 . This can be explained by the increase of the hardness. The Vickers microhardness measured under $20 \mathrm{~g}$ load is also presented in table 1 . The well-annealed aluminium sample has the lower microhadness, i.e., about $16 \mathrm{HV}$; however, the reference aluminium sample exhibits the higher microhardness of about $28.6 \mathrm{HV}$. The presence of the defects that occur 


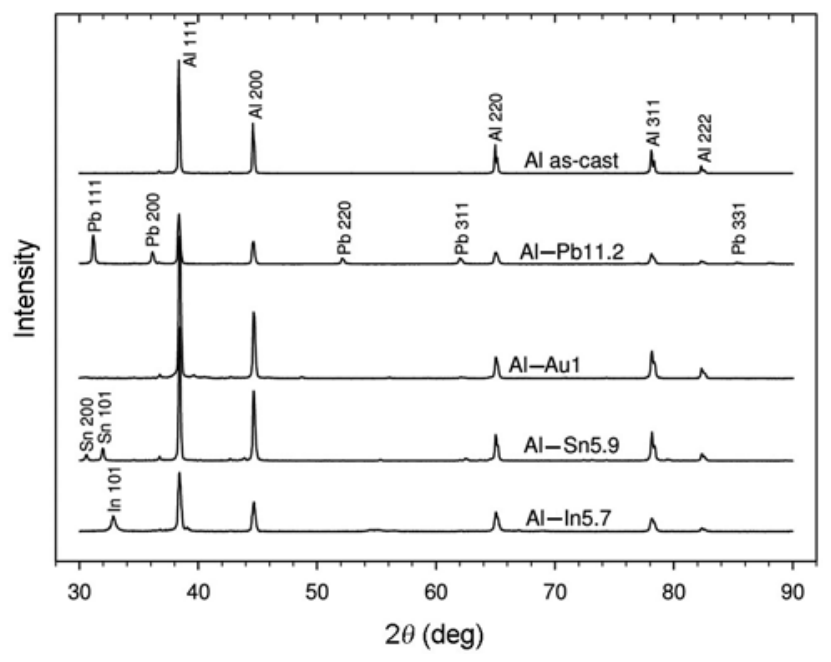

Figure 4. XRD pattern for the studied alloys.

during rapid solidification can explain the increase of the microhardness. Nevertheless, for the alloys the microhardness increases significantly. For the Al-In5.7 alloy, the obtained microhardness is about $71.6 \mathrm{HV}$ and it is higher by the factor of about 2.5 of the reference sample. Also the high value of the microhardness was obtained for the Al-Aul alloy. The correlation between the specific wear rate and micorhardness is found, i.e., the increase of the microhardness causes the increase of the wear resistance, it means the reduction of the specific wear rate. This is a well-known correlation. We can conclude that presence of the nanoparticles improves the mechanical properties of the alloys.

\subsection{Positron measurements}

It is reported that the positron lifetime in bulk, i.e., in perfect lattice in aluminium, is equal to 165 ps. For positrons trapped at the monovacancy in a perfect lattice it equals to $245 \mathrm{ps,}$ while for positrons trapped at the monovacancy located at edge dislocation is equal to about 225 ps. $^{16}$ Vacancy clusters are also suitable positron traps, in this case the positron lifetime is higher than $245 \mathrm{ps}$, and lower than $500 \mathrm{ps}$, and it depends on the number of vacancies in the cluster. ${ }^{17,18}$ These values are helpful for the identification of the type of defects present in a sample.

Positron lifetime spectra were measured for the raw alloys after preparation. In all spectra, single lifetime component was resolved and its values are given in table 1 . For the reference sample, the obtained lifetime is $229.7 \pm 0.5 \mathrm{ps}$ and this indicates the presence of the dislocations with vacancies close or at their lines. Similar value is detected for the Al-Aul alloy, for which the lifetime is about $224.1 \pm 0.5$. However, for the other alloys the obtained lifetime values are higher, e.g., for Al-In5.7 it is about $256.9 \pm 0.5$. This may suggest the presence of monovacancies or their cluster that consists of two vacancies in alloys. ${ }^{18}$

Certainly, positrons implanted into the alloy penetrate and thermalize in the interior of the nanoparticles. The positron lifetime in bulk is equal to 196, 214, 204 and 116 ps for $\mathrm{In}, \mathrm{Sn}, \mathrm{Pb}$ and $\mathrm{Au}$, respectively. ${ }^{16}$ Thus it is much below the measured value, i.e., about $250 \mathrm{ps}$. The small size of the particles, average diameter is about $100 \mathrm{~nm}$, is comparable with the positron diffusion length in these metals. Thus we can suppose that positrons thermalized inside a particle can diffuse to the interface between particle and the matrix. Usually, the interface contains open volume defects which are perfect traps for positrons.

The localization of the positron traps can be deduced from the CDB spectra measured for all samples. The useful presentation of the obtained results is the curve being the ratio of the CDB spectrum for the alloy under consideration to the CDB spectrum for pure well-annealed aluminium, because it enhances the region of the annihilation with the core electrons. Such dependences are depicted in figure 5 . In figure $5 \mathrm{a}$, the ratio curves are presented for pure In, the reference Al as-cast and the Al-In5.7 alloys. The data presented in figure 5 are rough and no smoothing procedure was used. The horizontal line represents the dependency for pure well-annealed Al sample. The maximum around $514 \mathrm{keV}$ visible for In ratio curve is also reproduced for the Al-In5.7 alloy, but its height is much lower. This maximum results the annihilation with p-electrons of core region. This unambiguously suggests that the monovacancies or divacancies which trap positrons must be decorated by In atoms. They must be located at the interface between In particles and the Al matrix. Similar conclusions can be drawn after inspection of the ratio curves for other alloys, see figure 5 . It is interesting that also for the In-Au0.5 alloy the maximum at photon energy of $513.5 \mathrm{keV}$ is well recognized and it is attributed to the annihilation with Au core electrons. The height of the maximum can be correlated with the concentration of the decorated vacancies. In figure 5, the ratio curve for the as-cast reference Al sample is also depicted. No maximum is observed and the small decline between 514 and $516 \mathrm{keV}$ results the localization of positrons at dislocation jogs mentioned above. ${ }^{18}$

One can assume that the ratio dependency measured for the alloy is the weighted sum of the dependencies for the reference $\mathrm{Al}$ as-cast and metal being an alloy element

$$
N_{\text {alloy }}(E)=(1-\eta) N_{\text {Al as-cast }}(E)+\eta N_{\text {metal }}(E) .
$$

The only parameter is the fraction of positrons $\eta$, which annihilate with electrons of the metal. Indeed the sum can well reproduce the measured ratio dependency for all alloys. The dashed lines in figure 5 represent the best fit of relation (1) to the measured ratio curves. The only one adjustable parameter is the fraction $\eta$. Hugenschmidt 
(a)
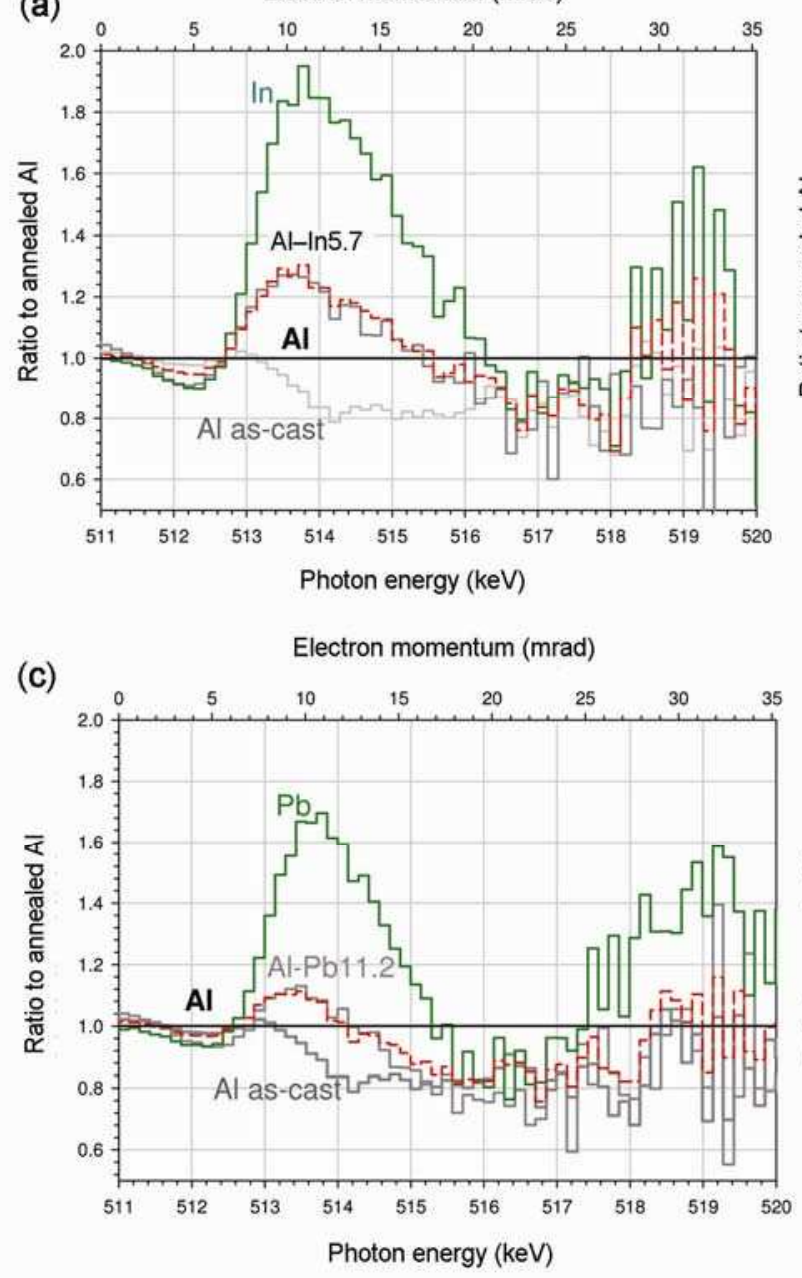

(b)
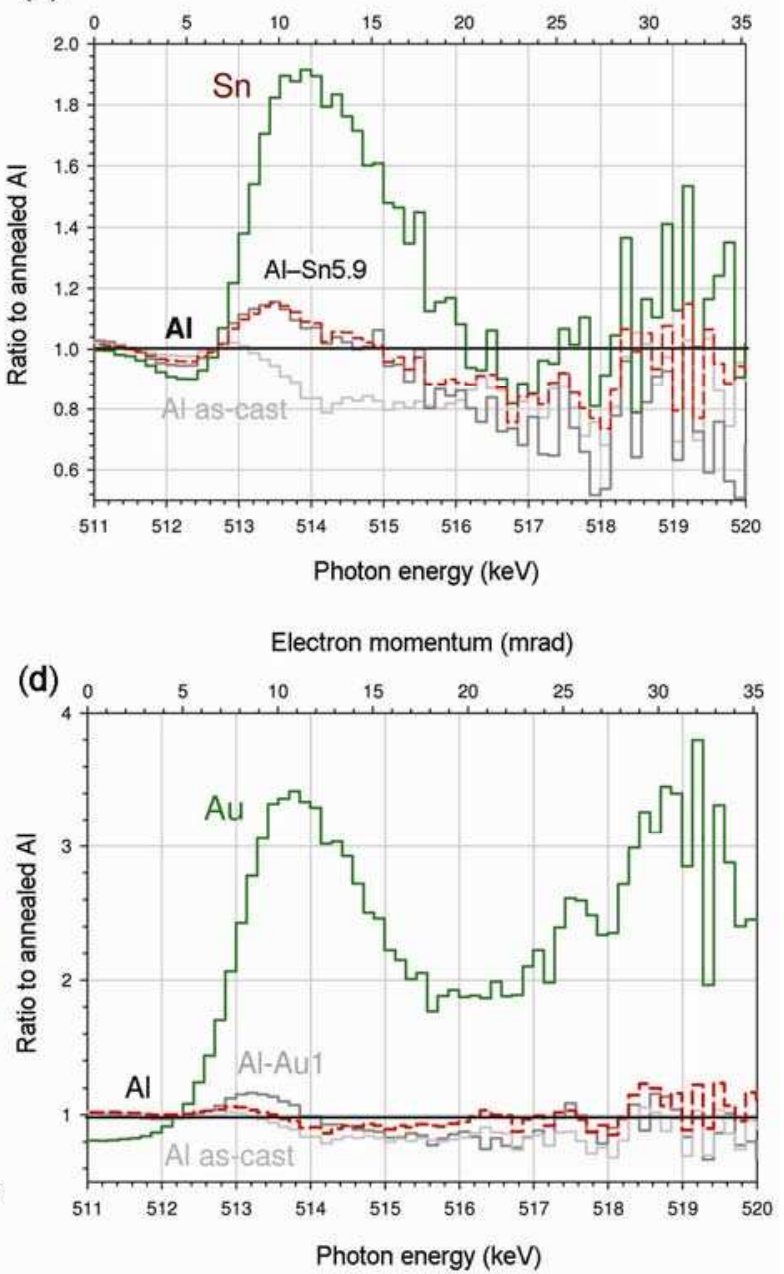

Figure 5. CDB spectra of the obtained alloys normalized to the spectrum of the as-cast reference Al. The dashed line represents the best fit of equation (1) to the measured CDB ratio curves for the alloys. The adjustable parameter represents the fraction of positrons $\eta$, which annihilate with electrons of the metal composing the embedded particles. Its values determined from the fit are as follows: 0.38, 0.23, 0.23 and 0.006 for the Al-In5.7, Al-Sn5.9, $\mathrm{Al}-\mathrm{Pb} 11.2$ and $\mathrm{Al}-\mathrm{Au} 1$ alloys, respectively.

et $a l^{11}$ and Pikart $e t a l^{12}$ propose to compare this parameter with the fraction of positrons implanted into the nanoparticles. This is reasonable because during random walk with the thermal energy, a positron localized in the nanoparticle can be used easily to find the interface and be trapped there. One should note that for layers and slow positrons used by these authors, it is simple to calculate theoretically the fraction of positron annihilating in the separate layers, but it is not for the particles randomly distributed. Nevertheless, it was assumed that the fraction $\eta$ can be compared with the volume fraction of the nanoparticles in the sample. For instance, for the Al-In5.7 alloy the volume fraction of nanoparticles calculated from the weight ratio is about 0.023 , but the value of $\eta$ obtained fitting equation (1) to the experimental is about one-order higher, i.e., 0.38. Similar increase is also observed for the Al-Sn5.9 alloys, the $\eta$-value determined from the fit for this alloy is: 0.23 and the corresponding volume fraction is equal to 0.023 . For the $\mathrm{Al}-\mathrm{Pb} 11.2$ and $\mathrm{Al}-\mathrm{Au} 1$ alloys the tendency is similar. This suggests the high positron affinity or the preferential localization to the nanoparticles of the studied alloys. In other words, the nanoparticles act as traps for positrons; if they jump into them they cannot escape to the matrix. Differences in internal positron and electron chemical potentials between the matrix and nanoparticles are the physical reason of this phenomenon. ${ }^{17}$ However, the complete discussion of this issue is beyond the scope of this paper. Nevertheless, the results reported in refs 11 and 12 obtained for layered samples are confirmed.

\subsection{Temperature measurements}

The alloys obtained by rapid solidification are in nonequilibrium state. This is indicated for instance by the 


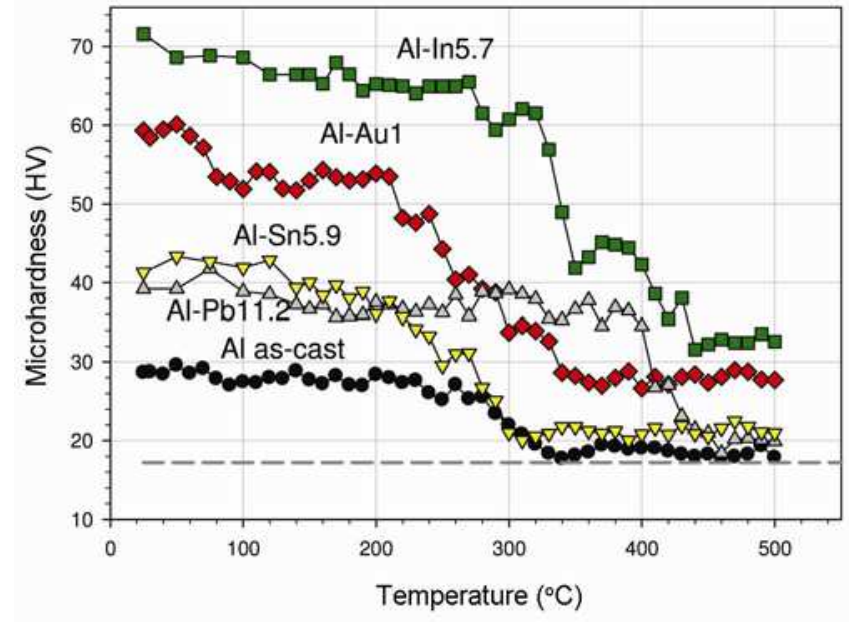

Figure 6. Microhardness as a function of the annealing temperature for the studied alloys.

fact that measured positron lifetime values are higher than the bulk value. The value of the positrons lifetime about $220 \mathrm{ps}$ is also obtained for aluminium sample exposed to deep plastic deformation. The calorimetric studies supplemented by measurements of electrical resisitivity and hardness performed by Clareborough et $a l^{19}$ for pure aluminium deformed at room temperature revealed two stages during annealing. The first stage is characterized by the broad peak at $100-250^{\circ} \mathrm{C}$, which corresponds to the heat evolved during dislocation recovery and the peak at $300^{\circ} \mathrm{C}$ that evolved during recrystallization. We can expect similar behaviour in the alloys studied by us. Moreover, the presence of defects, mainly dislocations and their loops causes the hardening of the alloys. The removal of defects during recovery and recrystallization induced by the temperature increase can reduce the hardness. Our samples were subjected to cumulative isochronal annealing in the flow of air for $1 \mathrm{~h}$ in the temperature range between 50 and $500^{\circ} \mathrm{C}$ with temperature step of $10^{\circ} \mathrm{C}$. In figure 6 , the dependency of the microhardness on the annealing temperature is depicted. It can be noticed that for the as-cast $\mathrm{Al}$ (closed circles) the microhardness remains constant up to the temperature of $250^{\circ} \mathrm{C}$, then in the temperature range from 250 to $350^{\circ} \mathrm{C}$ it decreases. Above $350^{\circ} \mathrm{C}$ the microhardness also remains constant but at a lower value. Similar dependencies are obtained for other studied alloys; however, the temperature range where the transition to the lower value of the microhardness takes place is slightly shifted towards higher temperatures. For instance, for Al-In5.7 alloy the decrease starts at $370^{\circ} \mathrm{C}$ and finishes at $430^{\circ} \mathrm{C}$. This indicates that the process of recrystallization occurred, and new grains grew free of defects.

The confirmation of this can be found in the temperature dependency of the $S$-parameter, depicted in figure 7. The value of the $S$-parameter is sensitive to the presence of open volume defects, which accompanies dislocation
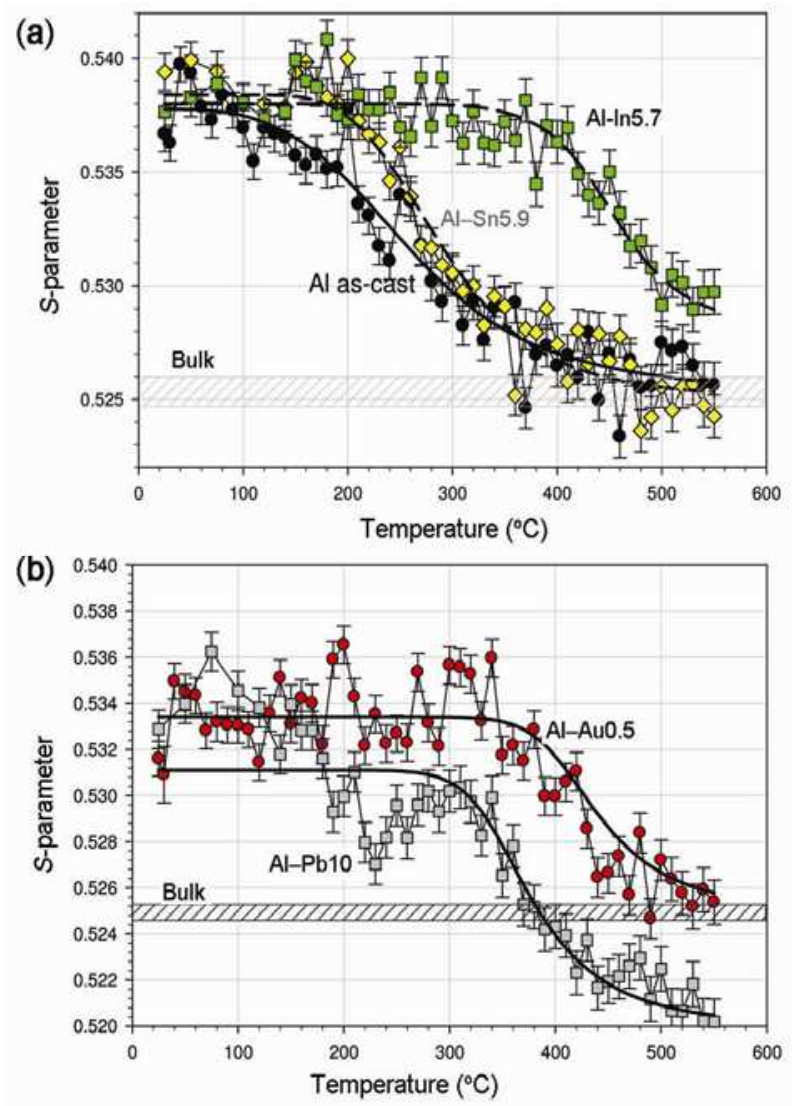

Figure 7. $S$-parameter $v s$. the annealing temperature for the studied alloys. The solid line represents the best fit of the dependency obtained from the diffusion trapping model. The main parameter obtained from the fit is the activation energy for grain boundary migration $Q$. The values obtained for the alloys studied are gathered in table 1.

structure; the decrease of their concentration is reflected in the decrease of the $S$-parameter value. The decrease of the $S$-parameter value to the bulk value, tagged in figure 7 as the hatched region at the temperature of $400^{\circ} \mathrm{C}$ for the as-cast $\mathrm{Al}$, indicates the removal of defects and nucleation and growth of new grains free of defects. It can be noticed that transition range is slightly larger, for instance for the as-cast $\mathrm{Al}$ it is between 150 and $400^{\circ} \mathrm{C}$ in comparison to the microhardness. This can arise from the much higher sensitivity of positrons to defects in comparison to microhardness measurements. Similar dependencies are obtained for the alloys as shown in figure 7 , however the transition range is shifted towards slightly higher temperatures.

In our previous studies it was found that the so-called diffusion trapping model can be applied with success for description of the temperature dependency of the $S$ parameter when the recrystallization process in a sample takes place. ${ }^{20}$ This model takes into account: the positron annihilation in the defect-free grain where additionally the positron can diffuse, trapping and annihilation at the grain boundary and kinetics of the grain grew. This is 
necessary because the temperature increases cause grain boundaries migration and the grain size increase. Due to low value of the positron diffusion length, about $0.1 \mu \mathrm{m}$, only the initial stage of recrystallization can be traced. When grains have the radius more than few micrometres, the trapping and annihilation at grain boundaries can be neglected in this model. The detailed description of the model is given in ref. 20. The solid curve presents the best fit of the model to the dependency in figure 7 . The important parameter in the model is activation energy of boundary migration of new grains. This is the adjustable parameter that can be determined in the fitting procedures. For as-cast Al, this parameter determined is equal to $0.63 \pm 0.1 \mathrm{eV}$. In the literature, activation energy for boundary diffusion in aluminium is equal to $0.87 \mathrm{eV} .^{21,22}$ For other alloys the activation energy is higher. For instance, the highest value is achieved for the Al-In5.7 alloy, i.e., $2.12 \pm 0.4 \mathrm{eV}$. For other alloys these values are gathered in table 1. The other adjustable parameters in the model are as follows: the $S$-parameter for grain boundary and inside the grain and the parameter related to the transition rate of positrons from the grain to the boundary. The $S$-parameter and microhardness measurements allow us to draw the conclusion that the nanoparticles immersed in the matrix prevent the grain boundary migration. However, as it was claimed by Gottstein et $a l^{23}$ the activation energy in aluminium depends on many factors.

\section{Conclusions}

The nanoparticles embedded in the studied aluminium alloys significantly increase hardness and also improve frictional properties in comparison to the as-cast pure aluminium sample. The reduction of the specific wear rate and friction coefficient is well demonstrated. However, the presence of point defects in these alloys is also observed. For the as-cast reference $\mathrm{Al}$ and the $\mathrm{Al}-\mathrm{Aul}$ alloys the presence of dislocations is revealed, whereas in the other studied alloys mainly monovacancies or divacancy clusters at the interface between the nanoparticles were detected. It seems that these defects affect significantly the mechanical properties of these alloys. The defects are removed during annealing in the alloys as the result of the recovery and recrystallization. These processes occur in the temperature range between 200 and $500^{\circ} \mathrm{C}$ depending on the alloy. The presented studies confirmed the attractive positron affinity of the nanoparticles of In, $\mathrm{Sn}, \mathrm{Pb}$ and $\mathrm{Au}$ compared to the Al matrix.

\section{Acknowledgement}

This research project has been partly supported by the European Commission under the 7th Framework Programme through the 'Research Infrastructures' action of the 'Capacities' Programme, NMI3-II Grant number 283883.

\section{References}

1. Forrester P G 1960 Met. Rev. 5507

2. Pathak J P and Mohan S 2001 Bull. Mater. Sci. 26315

3. Bhattacharya V and Chattopadhyay K 2001 Scr. Mater. 44 1677

4. Bhattacharya $\mathrm{P}$, Bhattacharya $\mathrm{V}$ and Chattopadhyay $\mathrm{K}$ 2002 J. Mater. Res. 172875

5. Bhattacharya V and Chattopadhyay K 2004 Acta Mater. 52 2293

6. Bhattacharya V and Chattopadhyay K 2004 Mater. Sci. Eng. A375-377 932

7. Yuan L, Jing G, Yarrqing S, Hong-Sheng A and Jun J 2001 Trans. Nonferrous Met. Soc. China 1184

8. Bhattacharya $\mathrm{P}$, Bhattacharya V and Chattopadhyay $\mathrm{K}$ 2002 J. Mater. Res. 172875

9. Krause-Rehberg R and Leipner H S 1999 In Positron annihilation in semiconductors, defect studies (Berlin, Heidelberg, New York, Hong Kong, London, Milan, Paris, Tokyo: Springer)

10. Dupasquier A, Kögel G and Somoza A 2004 Acta Mater. 524707

11. Hugenschmidt C, Pikart P, Stadlbauer M and Schreckenbach K 2008 Phys. Rev. B 77092105

12. Pikart P, Hugenschmidt $\mathrm{C}$ and Horisberger A 2011 Phys. Rev. B 84014106

13. Dryzek J and Singleton D 2006 Nucl. Instrum. Methods Phys. Res. B252 197

14. See: http://www.ifj.edu.pl/ mdryzek/index.html

15. See http://imagej.nih.gov/ij/

16. Campillo Robles J M, Ogando E and Plazaola F J 2007 J. Phys.: Condens. Matter 19176222

17. Puska M J, Lanki P and Nieminen R M 1989 J. Phys. Condens. Matter. 16081

18. Luna C R, Macchi C, Juan A and Somoza A 2013 J. Phys. Conf. Ser. 443012019

19. Clareborough L M, Hargreaves M E and Loretto M H 1963 Recovery and recrystallization of metals (Himmel, Interscience)

20. Dryzek J 2014 Appl. Phys. A 114465

21. Humphreys F J and Hatherly M 2004 Recrystallization and related annealing phenomena (Amsterdam: Elsevier)

22. Frost H J and Ashby M F 1982 Deformation-mechanism maps (Pergamon Press)

23. Gottstein G, Molodov D A, Czubayko U and Shvindlerman L S 1995 J. Phys. IV 589 